\title{
Optimization and Prediction of Melting Efficiency of Mild Steel Weldment, Using Genetic Algorithm
}

\author{
Sibete. $\mathbf{G}^{1}$, Eyitemi. $\mathbf{T}^{2}$ \\ ${ }^{1}$ Department of Mechanical Engineering, Niger Delta University, Wilberforce Island, Bayelsa State, Nigeria. \\ ${ }^{2}$ Department of Mechanical Engineering, Nigeria Maritime University, Okerenkoko ,Delta State, Nigeria.
}

Corresponding Author: Godfrey Sibete

\begin{abstract}
Melting efficiency which indicates how much of the heat deposited by the welding operation is used to produce melting is one of the most important parameters considered in Tungsten Inert Gas (TIG) welding when assessing the performance of welds. In the field of welding, a good melting efficiency results in the development of a dense weld pool. This study is conducted to optimize and predict the melting efficiency of mild steel weldment, using Genetic Algorithm. Genetic Algorithm (GA), which is an optimization method that mimics the evolution process and operates on the basis of the theory of natural selection and evolution was used to analyse the results. The result shows that a combination of current $239.03 \mathrm{~A}$, voltage $29.87 \mathrm{~V}$, welding speed $56.59 \mathrm{~mm} / \mathrm{s}$, welding time $79.15 \mathrm{sec}$, feed rate $130 \mathrm{~mm} / \mathrm{s}$, will produce optimal melting efficiency of 44.72 .
\end{abstract}

Keywords: Melting Efficiency, Mild Steel Weldment, Genetic Algorithm, Optimization and Prediction

\section{INTRODUCTION AND LITERATURE REVIEW}

The TIG welding process is extensively used in modern industrial manufacturing, this process is known for its economy, ability to join similar and dissimilar metals at very high temperature, low heat affected zone, absence of slag, good weld appearance, high quality weld, high heat concentration with little or no smoke or fumes made it suitable for both ferrous metals. Basically, TIG weld quality is strongly distinguished by the weld bead geometry.

Urena et al (2014) investigated the influence of the interface transformation between the $\mathrm{Al}$ alloy matrix and $\mathrm{SiC}$ particle reinforcement on the rupture behaviour in TIG welded Al matrix composites. TIG welding was performed on AA2014/SiC/Xp sheets of $4 \mathrm{~mm}$ thickness utilizing current settings in the range of 37-155 $\mathrm{A}$ and voltage of 14-16.7 V. It was discovered from the experimental results that, the failure happened in the weld metal with a tensile strength that is lower than $50 \%$ of the parent material. Fracture of the joint welded was controlled by interface deboning through the interface reaction Layer. Probability of interface failure rises in the weld zone due to formation of Aluminiumcarbide which lowers the matrix/ reinforcement interface strength.

Simhachalam et al (2015) carried out studies on the influence of welding operation parameters on the mechanical properties of stainless steel $-316(18 \mathrm{Cr}-8 \mathrm{~N})$ welded by TIG welding. The size of the specimen is $40 \times 15 \times 5 \mathrm{~mm}$ for the experiment discovered that the current has a very important effect, though the filler rod also have some influence which is also similar to the current but when compared to current it is less important. MINITAB software is employed for the prediction of the depth of penetration, hardness and impact strength.

Sanjeev (2016) did the experiment for the optimization of the condition for performing the welding on Utra-90 
Sibete. G et.al. Optimization and prediction of melting efficiency of mild steel weldment, using genetic algorithm.

specimen where he alters the current and the voltage while the gas flow rate is kept constant and discovered that the welding joint which is not made properly below $50 \mathrm{~A}$ and above 200A, from there the burning of specimen started.

Ravinder(2016) researched the Optimization of the parameters of TIG welding of stainless steel (202) with mild steel by utilizing Taguchi method and discovered the control factor that had changing influence on the tensile strength, the arc voltage which have the highest influence and also discovered the optimum parameter for the tensile strength, current 80 A, Arc voltage $30 \mathrm{~V}$ and GFR 6 lt/min.

\section{METHODOLOGY AND THEORY}

The method of achieving the objectives of the research is explained in this chapter.

\subsection{Using Genetic Algorithm}

1. How the genetic algorithm functions

2. Outline of the algorithm

a. The algorithm starts by forming a random start population.

b. Then the algorithm forms a sequence of new populations. At every step, the algorithm utilizes the individuals in the currents generations to form the new population. To form the new population, the algorithm does the following steps below.

c. Gives score to every member of the present population by calculating its fitness value.

d. Weighs the raw fitness scores to change them into a more useful range of values.

e. Members are identified as parents, on the basis of their fitness.

f. Certain individuals in present population having lower fitness are then selected as elite. These elite individuals are moved on to the next population.

g. forms children from the parents. Children are formed by either random alterations to a single parent-mutation-or by combination of the vector entries of a pair parents-crossover. h. Replace the present population and the children to produce the next generation

\subsection{GENETIC ALGORITHM}

Let $\mathrm{X}_{1}, \mathrm{X}_{2}, \mathrm{X}_{3}, \mathrm{X}_{4}$ and $\mathrm{X}_{5}$ represent current, voltage, speed, time and feed rate respectively; $f(x)$ the vector of fitness functions.

The optimization problem becomes

$\operatorname{Min} \mathbf{f}(\mathbf{x})$, subject to

$$
\left[\begin{array}{l}
X_{1} \\
X_{2} \\
X_{3} \\
X_{4} \\
X_{5}
\end{array}\right] \geq 0 ;\left[\begin{array}{c}
160 \\
20 \\
35 \\
50 \\
70
\end{array}\right] \leq\left[\begin{array}{l}
X_{1} \\
X_{2} \\
X_{3} \\
X_{4} \\
X_{5}
\end{array}\right] \leq\left[\begin{array}{c}
240 \\
30 \\
75 \\
80 \\
140
\end{array}\right]
$$

The components of the fitness function, $\mathrm{f}(\mathrm{x})$ are

$\mathrm{f}_{1}=1438+20.6 \mathrm{X}_{1}+13.3 \mathrm{X}_{2}-8.2 \mathrm{X}_{3}+$ $11.4 \mathrm{X}_{4}+11.2 \mathrm{X}_{5}+0.043 \mathrm{X}_{1} \mathrm{X}_{2}-0.047 \mathrm{X}_{1} \mathrm{X}_{5}-$ $0.32 \mathrm{X}_{2} \mathrm{X}_{4}-0.045 \mathrm{X}_{4} \mathrm{X}_{5}-0.0458 \mathrm{X}_{1}^{2}$

$\mathrm{f}_{2}=653+11.9 \quad \mathrm{X}_{1}-5.9 \mathrm{X}_{2}-8.25 \quad \mathrm{X}_{3}-3.38$

$\mathrm{X}_{4}+6.582 \mathrm{X}_{5}+0.032 \quad \mathrm{X}_{1} \mathrm{X}_{5}+0.033 \mathrm{X}_{3} \mathrm{X}_{4}$ $0.03 \mathrm{X}_{1}^{2}$

$\mathrm{f}_{3}=41+0.092 \mathrm{X}_{1}-0.52 \mathrm{X}_{2}-0.09 \mathrm{X}_{3}+0.39 \mathrm{X}_{4}-$ $0.14 \mathrm{X}_{5}+\quad 0.00072 \mathrm{X}_{1} \mathrm{X}_{3}-$ $0.0021 \mathrm{X}_{1} \mathrm{X}_{4}+0.0046 \mathrm{X}_{2} \mathrm{X}_{5}+0.00069 \mathrm{X}_{3} \mathrm{X}_{5}-$ $0.0013 \mathrm{X}_{3}^{2}$

$\mathrm{f}_{4} \quad=$

$74.124+0.3663 * X_{1}+2.6655 X_{2}+1.6834 X_{3}+0$. $019708 X_{1} X_{2}-0.0079 X_{1} X_{3}-0.06204 X_{2} X_{3}$

$$
\left[\begin{array}{l}
f_{1} \\
f_{2} \\
f_{3} \\
f_{4}
\end{array}\right]=\left[\begin{array}{c}
T_{s} \\
T_{m} \\
\eta \\
H I
\end{array}\right]
$$

All but the melting efficiency are to be minimized. Since we desire to maximize the melting efficiency, $\eta$, we therefore minimize $-\mathrm{f}_{3}$

The following options along with the fitness function were fed into the genetic algorithm toolbox in MatLab software

Number of variables: 5

Population type: Double vector

Population size: $75 \quad\left(15^{*}\right.$ Number of variable)

Creation function: Feasible Constraint dependent 
Sibete. G et.al. Optimization and prediction of melting efficiency of mild steel weldment, using genetic algorithm.

Initial population: Default (created using the fitness function)

Initial score: Default

Initial range: Default $-[0,1]$

Selection function: Tournament

Tournament size: 2

Crossover fraction: 0.8

Mutation function: Constraint dependent

Crossover function; Scattered

Migration fraction: 0.2

Migration Direction: Both

Migration Interval: 20

Stopping Criteria
- Generations: 1000 (200* no of variables)

- Time limit: Infinite

- Fitness limit: Infinite

- Stall generations: 100

- Function tolerance: 0.0004

An initial population of seventy five (75) individuals was generated along with the associated Score values as shown in table 4.31 .

Table 4.31 Population and Score

\begin{tabular}{|c|c|c|c|c|c|c|c|c|c|}
\hline & \multicolumn{5}{|l|}{ Popula } & \multicolumn{4}{|l|}{ Score } \\
\hline & I & $\mathbf{V}$ & V & $\mathbf{T}$ & FR & $\mathbf{T}_{\mathrm{s}}$ & $\mathbf{T}_{\mathrm{m}}$ & $\eta$ & HI \\
\hline 1 & 240.00 & 30.00 & 35.03 & 79.98 & 139.99 & 1155.40 & 3570 & 43.57 & 163.0 \\
\hline 2 & 0.00 & 30.00 & 35.02 & 79.19 & 139.98 & 58.94 & 3572.13 & 43.65 & 63.03 \\
\hline 3 & 5.39 & 29.98 & 74.98 & 78.21 & 70.09 & 234.76 & 2141.56 & 3.07 & 52.90 \\
\hline 4 & 0.00 & 0.00 & 35.02 & 79.89 & 139.99 & 155.80 & 3570.52 & 43.58 & 163.03 \\
\hline 5 & 65.02 & 20.71 & 64.07 & 77.15 & 109.87 & 386.84 & 2674.67 & 45.90 & 50.90 \\
\hline 6 & 0.00 & 30. & 35.03 & 79.97 & & +3 & 357 & 43.57 & \\
\hline 7 & 9.17 & & 52.52 & 79.53 & & & 336 & .35 & \\
\hline 8 & 9.03 & 29.87 & 56.59 & 79.15 & 130.99 & 230.89 & 3432.46 & 4.72 & 17.30 \\
\hline 9 & 0.00 & 30.00 & 35.01 & 79.17 & 139.54 & 160.07 & 3565.80 & 3.65 & 163.06 \\
\hline 10 & 164.14 & 20.00 & 75.00 & 70.22 & 116.30 & 386.17 & 2733.49 & 44.83 & 39.96 \\
\hline 11 & 165.37 & 29.98 & 74.99 & 78.22 & 70.10 & 1234.61 & 2141.46 & 43.07 & 52.87 \\
\hline 12 & 165.37 & 29.98 & 74.99 & 78.22 & 70.11 & 1234.62 & 2141.48 & 43.07 & 52.87 \\
\hline 13 & 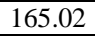 & 20 & 64.07 & 77.15 & & 84 & 2674.67 & 5.90 & 0.90 \\
\hline 14 & 164.16 & 21 & 75.00 & 70.22 & 116.30 & 386.23 & 27. & 4.83 & 9.90 \\
\hline 15 & 5.39 & 2 & 74.98 & 78.21 & 70.09 & 77 & 56 & 3.07 & 52.89 \\
\hline 16 & 5.48 & 10 & 74.96 & 78.21 & 70.09 & 28 & 2142.26 & 3.07 & 52.95 \\
\hline 17 & 9.02 & 29.90 & 68.82 & 79.34 & 87.01 & 356.26 & 2801.65 & 2.90 & 92.23 \\
\hline 18 & 40.00 & 30.00 & 35.02 & 79.89 & & 1155.80 & & 43.58 & 163.03 \\
\hline 19 & & 30 & 35. & 79. & & & & 3.65 & \\
\hline 20 & & 29. & 42.19 & 79.47 & & & & .04 & \\
\hline 21 & 0.00 & 3 & 35.02 & 79. & 13 & 71 & 35 & 3.58 & \\
\hline 22 & 3.56 & 2 & 56.73 & 79.09 & & 90 & 12 & .06 & 07.90 \\
\hline 23 & 99.99 & 26. & 39.47 & 72.81 & 140.00 & 91 & 58 & 4.22 & 134.73 \\
\hline 24 & 5.37 & 29.98 & 74.99 & 78.22 & 70 & 61 & & 3.07 & 2.87 \\
\hline 25 & 772 & 20.31 & 74.52 & 79.75 & & & & 3.93 & 2.02 \\
\hline 26 & & 29 & 52.5 & 79. & & & & 4.35 & \\
\hline 27 & 9.95 & 3 & 35.26 & 79. & & 65 & & 2.86 & .48 \\
\hline 28 & 1 & 2 & 75.00 & 77.06 & & 16 & & 5.05 & 10 \\
\hline 29 & 165.45 & 2 & 74.97 & 78.21 & 70 & 10 & 2142.12 & 3.07 & 52.93 \\
\hline 30 & 165.39 & 29. & 74.97 & 78.21 & 70. & 4.79 & 141.62 & 3.07 & 52.92 \\
\hline 31 & ( & 27. & 35.87 & 73.91 & & & 359 & 43.92 & 146.26 \\
\hline 32 & 1 & 29. & 74.89 & 79.92 & & & & 42.66 & 3.63 \\
\hline 33 & & & 35 & & & & & 82 & 160.69 \\
\hline 34 & & 23 & 72 & 77. & & & & .21 & 48.44 \\
\hline 35 & 1.70 & 29.98 & 74.72 & 78.80 & 71. & 398.85 & 2462.64 & 2.43 & 70.28 \\
\hline 36 & 9.14 & 29.96 & 42.17 & 79.31 & 93.63 & 285.61 & 2905.67 & 43.01 & 147.49 \\
\hline 37 & 234.17 & 29.45 & 48.25 & 77.47 & 74.17 & 362.12 & 2617.43 & 3.04 & 129.88 \\
\hline 38 & 195.86 & 29.80 & 73.13 & 79.65 & & 366.42 & 2361.89 & 42.81 & 6.83 \\
\hline 39 & & 29. & 64.95 & 79. & & 69 & & 42.70 & 100.88 \\
\hline 40 & & 29 & .70 & 79 & & & & 69 & \\
\hline 41 & & & & & & & & 44.44 & 139.95 \\
\hline 42 & 9 & 29. & 15 & 78.30 & 77 & 375.36 & 26 & 2.77 & 100.76 \\
\hline 43 & 7.64 & 29.52 & 61.02 & 78.76 & 77. & 1370.69 & 2664.74 & 42.84 & 106.28 \\
\hline 44 & 5.37 & 29.98 & 74.99 & 78.22 & 70 & 234.62 & 2141.48 & 43.07 & 52.87 \\
\hline 45 & 8.48 & 29.62 & 3.68 & 79.94 & 71. & 06.23 & & 1.95 & 81.23 \\
\hline 46 & 2 & 29. & 42.80 & 79.71 & (5) & 21.83 & .70 & 43.70 & 46.19 \\
\hline 47 & & 30. & 07.57 & & & & & 27 & 45 \\
\hline 48 & & 29 & 74.97 & 78.21 & & 10 & 21 & 43.07 & 52.93 \\
\hline 49 & 165.37 & 29.98 & 74.99 & 78.22 & 70.10 & 1234.61 & 2141.46 & 43.07 & 52.87 \\
\hline
\end{tabular}


Sibete. G et.al. Optimization and prediction of melting efficiency of mild steel weldment, using genetic algorithm.

\begin{tabular}{|l|l|l|l|l|l|l|l|l|l|}
\hline \multicolumn{10}{|c|}{ Table 4.31 Continued... } \\
\hline 50 & 237.30 & 28.69 & 71.67 & 79.78 & 71.47 & 1414.02 & 2576.05 & 42.29 & 82.18 \\
\hline 51 & 164.16 & 20.00 & 75.00 & 70.22 & 116.30 & 1386.24 & 2733.66 & 44.83 & 39.96 \\
\hline 52 & 228.14 & 29.74 & 74.51 & 79.68 & 70.47 & 1413.55 & 2520.47 & 42.07 & 76.11 \\
\hline 53 & 193.04 & 20.02 & 70.51 & 71.61 & 129.10 & 1459.66 & 3145.58 & 45.10 & 49.70 \\
\hline 54 & 239.99 & 29.68 & 36.03 & 77.15 & 139.99 & 1173.83 & 3577.85 & 43.92 & 159.28 \\
\hline 55 & 164.72 & 29.92 & 75.00 & 72.34 & 80.56 & 1250.98 & 2274.68 & 43.31 & 52.56 \\
\hline 56 & 239.87 & 28.84 & 38.69 & 74.19 & 134.32 & 1213.56 & 3505.61 & 44.24 & 149.56 \\
\hline 57 & 233.15 & 29.97 & 70.16 & 78.28 & 74.03 & 1396.29 & 2596.24 & 42.47 & 87.31 \\
\hline 58 & 218.36 & 28.70 & 71.69 & 79.17 & 70.86 & 1418.95 & 2488.65 & 42.70 & 75.24 \\
\hline 59 & 240.00 & 30.00 & 35.03 & 79.75 & 139.58 & 1157.45 & 3565.05 & 43.59 & 163.01 \\
\hline 60 & 238.61 & 29.95 & 58.48 & 79.64 & 76.65 & 1358.86 & 2655.76 & 42.67 & 113.51 \\
\hline 61 & 239.95 & 29.99 & 38.51 & 79.97 & 138.02 & 1167.70 & 3540.98 & 43.83 & 155.72 \\
\hline 62 & 239.64 & 29.98 & 61.38 & 79.65 & 82.76 & 1348.06 & 2745.11 & 42.83 & 108.13 \\
\hline 63 & 180.00 & 29.26 & 74.98 & 79.16 & 70.47 & 1316.18 & 2252.82 & 43.02 & 57.09 \\
\hline 64 & 240.00 & 30.00 & 35.03 & 79.99 & 139.99 & 1155.37 & 3570.34 & 43.57 & 163.01 \\
\hline 65 & 240.00 & 30.00 & 35.02 & 79.89 & 139.99 & 1155.80 & 3570.52 & 43.58 & 163.03 \\
\hline 66 & 165.45 & 29.98 & 74.97 & 78.21 & 70.10 & 1235.11 & 2142.12 & 43.07 & 52.93 \\
\hline 67 & 240.00 & 30.00 & 35.03 & 79.99 & 139.99 & 1155.38 & 3570.36 & 43.57 & 163.01 \\
\hline 68 & 240.00 & 30.00 & 35.02 & 79.19 & 139.98 & 1158.94 & 3572.13 & 43.65 & 163.03 \\
\hline 69 & 240.00 & 30.00 & 35.01 & 79.42 & 139.54 & 1158.95 & 3565.21 & 43.62 & 163.06 \\
\hline 70 & 238.47 & 30.00 & 75.00 & 79.69 & 70.45 & 1405.98 & 2560.59 & 41.80 & 79.57 \\
\hline 71 & 240.00 & 30.00 & 35.03 & 79.98 & 139.99 & 1155.40 & 3570.38 & 43.57 & 163.01 \\
\hline 72 & 238.91 & 29.96 & 51.57 & 79.31 & 85.71 & 1323.70 & 2788.93 & 43.02 & 127.95 \\
\hline 73 & 240.00 & 30.00 & 35.01 & 79.16 & 139.54 & 1160.11 & 3565.86 & 43.65 & 163.06 \\
\hline 74 & 211.70 & 29.98 & 74.72 & 78.80 & 71.90 & 1398.85 & 2462.64 & 42.43 & 70.28 \\
\hline 75 & 239.99 & 26.15 & 39.47 & 72.81 & 140.00 & 1234.92 & 3599.58 & 44.22 & 134.73 \\
\hline
\end{tabular}

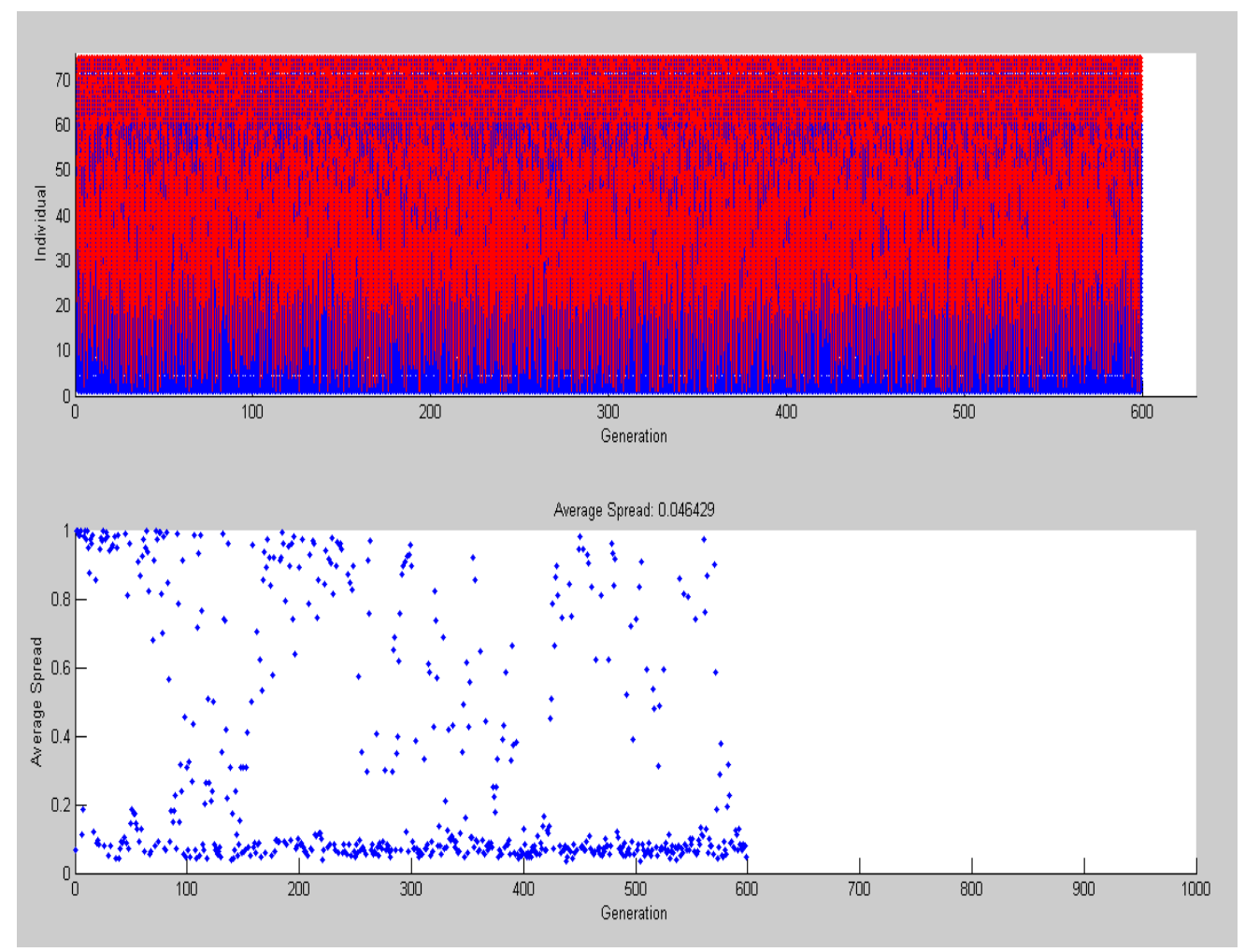

Fig 4.42 Plot of Genealogy and Average pareto spread

Fig 4.42 plots the genealogy of individuals. Lines from one generation to the next are color-coded as follows:

- Red lines indicate mutation children formed by making small random changes in the individuals in the population, which provide genetic diversity and enable the genetic algorithm to search a broader space

- Blue lines indicate crossover children which are formed by combining two individuals, or parents, to form a new individual, or child, for the next generation 
Sibete. G et.al. Optimization and prediction of melting efficiency of mild steel weldment, using genetic algorithm.

- Black lines indicate elite individuals which correspond to the individuals in the present generation with the best fitness values, the algorithm creates. These individuals automatically survive to the next generation
- Twenty seven (27) solutions were obtained from iterations over six hundred (600) generations.

The solutions are as shown in table 4.32 while the generations are shown in table 4.33

Table 4.32 Individuals and function values

\begin{tabular}{|l|l|l|l|l|l|l|l|l|l|}
\hline & $\mathbf{X}$ & $\mathbf{V}$ & $\mathbf{S}$ & $\mathbf{T}$ & $\mathbf{F}$ & $\mathbf{T}_{\mathbf{s}}$ & $\mathbf{T}_{\mathbf{m}}$ & $\mathbf{\eta}$ & $\mathbf{H I}$ \\
\cline { 2 - 11 } & $\mathbf{I}$ & $\mathbf{V}$ & $\mathbf{S}$ & $\mathbf{7}$ & $\mathbf{l}$ \\
\hline 1 & 239.03 & 29.87 & 56.59 & 79.15 & 130.99 & 1230.89 & 3432.46 & 44.72 & 117.30 \\
\hline 2 & 164.14 & 20.00 & 75.00 & 70.22 & 116.30 & 1386.17 & 2733.49 & 44.83 & 39.96 \\
\hline 3 & 239.02 & 29.90 & 68.82 & 79.34 & 87.01 & 1356.26 & 2801.65 & 42.90 & 92.23 \\
\hline 4 & 165.37 & 29.98 & 74.99 & 78.22 & 70.10 & 1234.61 & 2141.46 & 43.07 & 52.87 \\
\hline 5 & 239.95 & 30.00 & 35.26 & 79.55 & 105.00 & 1240.65 & 3072.22 & 42.86 & 162.48 \\
\hline 6 & 164.53 & 24.04 & 75.00 & 77.06 & 108.00 & 1333.16 & 2606.60 & 45.05 & 45.10 \\
\hline 7 & 240.00 & 27.10 & 35.87 & 73.91 & 140.00 & 1214.06 & 3594.71 & 43.92 & 146.26 \\
\hline 8 & 195.85 & 29.69 & 74.89 & 79.92 & 70.49 & 1367.86 & 2352.81 & 42.66 & 63.63 \\
\hline 9 & 239.03 & 30.00 & 35.83 & 79.68 & 100.49 & 1255.79 & 3004.33 & 42.82 & 160.69 \\
\hline 10 & 239.14 & 29.96 & 42.17 & 79.31 & 93.63 & 1285.61 & 2905.67 & 43.01 & 147.49 \\
\hline 11 & 195.86 & 29.80 & 73.13 & 79.65 & 70.93 & 1366.42 & 2361.89 & 42.81 & 66.83 \\
\hline 12 & 239.97 & 29.98 & 64.95 & 79.82 & 81.80 & 1356.69 & 2730.99 & 42.70 & 100.88 \\
\hline 13 & 236.72 & 29.91 & 46.90 & 79.15 & 74.16 & 1346.64 & 2620.33 & 42.69 & 136.09 \\
\hline 14 & 239.09 & 30.00 & 45.89 & 79.94 & 139.45 & 1184.22 & 3554.64 & 44.44 & 139.95 \\
\hline 15 & 239.76 & 29.88 & 42.80 & 79.71 & 120.35 & 1221.83 & 3287.70 & 43.70 & 146.19 \\
\hline 16 & 238.87 & 30.00 & 67.37 & 79.12 & 138.85 & 1234.09 & 3539.14 & 45.27 & 95.45 \\
\hline 17 & 228.14 & 29.74 & 74.51 & 79.68 & 70.47 & 1413.55 & 2520.47 & 42.07 & 76.11 \\
\hline 18 & 164.72 & 29.92 & 75.00 & 72.34 & 80.56 & 1250.98 & 2274.68 & 43.31 & 52.56 \\
\hline 19 & 233.15 & 29.97 & 70.16 & 78.28 & 74.03 & 1396.29 & 2596.24 & 42.47 & 87.31 \\
\hline 20 & 240.00 & 30.00 & 35.03 & 79.75 & 139.58 & 1157.45 & 3565.05 & 43.59 & 163.01 \\
\hline 21 & 238.61 & 29.95 & 58.48 & 79.64 & 76.65 & 1358.86 & 2655.76 & 42.67 & 113.51 \\
\hline 22 & 239.95 & 29.99 & 38.51 & 79.97 & 138.02 & 1167.70 & 3540.98 & 43.83 & 155.72 \\
\hline 23 & 239.64 & 29.98 & 61.38 & 79.65 & 82.76 & 1348.06 & 2745.11 & 42.83 & 108.13 \\
\hline 24 & 240.00 & 30.00 & 35.03 & 79.99 & 139.99 & 1155.37 & 3570.34 & 43.57 & 163.01 \\
\hline 25 & 238.47 & 30.00 & 75.00 & 79.69 & 70.45 & 1405.98 & 2560.59 & 41.80 & 79.57 \\
\hline 26 & 238.91 & 29.96 & 51.57 & 79.31 & 85.71 & 1323.70 & 2788.93 & 43.02 & 127.95 \\
\hline 27 & 211.70 & 29.98 & 74.72 & 78.80 & 71.90 & 1398.85 & 2462.64 & 42.43 & 70.28 \\
\hline
\end{tabular}

\section{RESULTS AND DISCUSSION}

In this study, Genetic Algorithm which is an optimization method that mimics the evolution process and operates on the basis of the theory of natural section and evolution was used to analyse the results. The result shows that a combination of current of $239.03 \mathrm{~A}$, voltage $29.87 \mathrm{~V}$, welding speed $56.59 \mathrm{~mm} / \mathrm{s}$, will produce optimal melting efficiency of 44.72

\section{CONCLUSION}

The integrity of a weld is ascertained by the quality of the weld bead geometry. Melting efficiency is a significant factor considered in assessing the integrity of the weld. In this study, an approach using the genetic algorithm for optimizing and predicting weld melting efficiency of mild steel weldment to improve the integrity of welded joints has been successfully introduced and its effectiveness and efficiency well demonstrated.

\section{Acknowledgement: None}

\section{Conflict of Interest: None}

\section{Source of Funding: None}

\section{REFERENCES}

1. Abhulimen, I.U. and Achebo, J.I. (2014): the utilization of adaptive neuro fuzzy interference system (ANFIS) in the weld output of a TIG welded pipe joint modelling. IMPACT: international journal of Engineering \& Technology research (IMPACT: LIRET) ISSN (E); 2321-8843; ISSN (P): 2347-4599 Vol.2, Issue 9, pp 99110.

2. Box.G. E. P and Draper. N. R. (1959). "A Foundation for the choice of a Response 
Sibete. G et.al. Optimization and prediction of melting efficiency of mild steel weldment, using genetic algorithm.

Surface Design Journal of the Statistical Association. . 54, 622-654.

3. Myers, R. H., Montgomery, D. C., \& Anderson-Cook, C. M. . Response Surface Methodology Product, Process Optimization utilizing Designed Experiments (Fourth ed.). Wiley,2016.

4. Myers, R.H, Khuri, A.I and Carter, W.H.(1989). Response Surface Methodology: 1966 -1988. Technometrics 31,137-157 MR1007291.

5. Myers, R.H. Montgomery, D.C and Anderson-Cook C.M, Response Surface Methodology operation and product optimization utilizing Designed
Experiments. John Wiley and Sons Pg 78, 2009.

6. S.P. Gadewar, PeravaliSwaminadhan, M. G. Harkare, weld characteristics experimental investigation for a single pass TIG welding with ss304 / Engineering Science and Technology, International Journal. Vol.2(8), 2010, 3676-3686.

How to cite this article: Sibete. G, Eyitemi. T. Optimization and prediction of melting efficiency of mild steel weldment, using genetic algorithm. International Journal of Research and Review. 2021; 8(6): 127-132. DOI: https://doi.org/10.52403/ijrr.20210615 